\title{
Whitening Dentifrices Effect on Enamel with Orthodontic Braces after Simulated Brushing
}

\author{
Vivian Santos Torres ${ }^{1}$ Max José Pimenta Lima² Heloísa Cristina Valdrighi ${ }^{1}$ Elisângela de Jesus Campos² \\ Milton Santamaria-J $\mathrm{r}^{1,3}$
}

${ }^{1}$ Graduate Program of Orthodontics, Hemínio Ometto

Foundation-FHO, Araras, São Paulo, Brazil

${ }^{2}$ Department of Biochemistry and Biophysics, UFBA, Salvador, Bahia, Brazil

${ }^{3}$ Graduate Program of Biomedical Sciences, Hemínio Ometto

Foundation-FHO, Araras, São Paulo, Brazil

\begin{abstract}
Address for correspondence Milton Santamaria-Jr, PhD, Centro Universitário da Fundação Hermínio Ometto-FHO/UNIARARAS, Avenida Maximiliano Baruto, Araras, São Paulo, Brazil, CEP 13607-339 (e-mail: santamariajr@ig.com.br).
\end{abstract}

Eur J Dent 2020;14:13-18

\begin{abstract}
Objective This study aimed to evaluate in vitro the effects of whitening dentifrices on enamel color, the shear bond strength of orthodontic brackets and adhesive remnant index (ARI).

Materials and Methods Eighty bovine teeth with brackets were randomly divided into four groups ( $n=20)$ : control group (GC)-water, test group 1 (GT1)-Colgate Total 12, test group 2 (GT2)-Curaprox Black Is White, and group test 3 (GT3)-Luminous White. All groups were submitted to brushing, simulating 12 months. The specimens were exposed to spectrophotometer color evaluation and to a shear strength test in a universal test machine using a $300 \mathrm{kN}$ load with a crosshead speed of $0.5 \mathrm{~mm} / \mathrm{min}$. The ARI was evaluated with a stereoscopic magnifying glass.

Statistical Analysis Nonparametric Kruskal-Wallis and Dunn's tests were used for the color analysis, and Friedman and Nemenyi tests were used to compare the times in the variable. To compare the shear force between the groups, the data were evaluated by one-way analysis of variance and Tukey's test, and ARI was analyzed using Fisher's exact test, always with a significance level of $5 \%$.

Keywords

- orthodontic brackets

- dentifrices

- tooth bleaching

- shear strength

Results In the color analysis, GT3 presented the greatest progression in whitening effect. GT1 had greater shear strength than GT3 did ( $p \leq 0.05$ ). For ARI, the score 1 was predominant in the GC and GT1. The GT2 and GT3 groups had scores of 3.

Conclusion The whitening dentifrices promoted significant color change over the 12-month brushing time and may have interfered in the resistance to shear bond strength and ARI.
\end{abstract}

\section{Introduction}

Aesthetic demands have increased among patients and interest in seeking procedures to provide better smile aesthetics, associated with the growing development of techniques and materials, has led to important advances in aesthetic dentistry. ${ }^{1}$ Although the terms "bleaching" and "whitening" are often used indiscriminately in dentistry, they are not synonymous. Bleaching is a process involving an oxidizing chemical that alters the absorption/reflection of light, increasing the perceived whiteness. ${ }^{2}$ Tooth bleaching is a process that results in whiter teeth and may include mechanical, chemical, and optical approaches that remove surface stains using abrasives and substances such as whitening dentifrices., ${ }^{2,3}$

The use of different types and concentrations of abrasives does not promote tooth whitening but is based on the mechanical or abrasive activity of removing biofilms and pigments adhered to the surface of tooth enamel, thus improving aesthetics and restoring the natural dental color. $^{4}$
DOI https://doi.org/ $10.1055 / \mathrm{s}-0039-3403474$ ISSN 1305-7456.
License terms

()(1) $\Theta \circledast$ 
Whitening dentifrices containing hydrated silica, calcium carbonate, dicalcium dihydrate phosphate, calcium pyrophosphate, alumina, perlite, or sodium bicarbonate mechanically remove biofilm stains on the surface of tooth enamel. In addition, daily use of these abrasives modifies the surface of the enamel by reducing biofilm adhesion, decreasing dental stains, and altering its color. ${ }^{5}$ Activated charcoal has attracted interest because it is present in some dentifrices, acting in superficial areas, and it has the ability to adsorb pigments and dyes responsible for changed tooth color. ${ }^{6}$

Factors such as smoking, consumption of foods, and/ or beverages containing pigments, use of products such as chlorhexidine and orthodontic treatments associated with toothbrush deficiency negatively influence smile aesthetics. Well-aligned white teeth show health and youth, so tooth whitening and orthodontic therapy are common treatments to promote beautiful smiles. ${ }^{7,8}$

The patient aesthetic expectation associated with orthodontic treatment has led the orthodontist to question the influence of whitening agents on brackets bond strength. Often the patients have desired to perform aesthetic treatments before and even during orthodontic therapy. ${ }^{9}$ Thus, this study evaluated the bond strength of the bonding and remnant adhesive of orthodontic brackets as well as the color change in bovine teeth submitted to simulated brushing with dentifrices containing bleaching and whitening agents.

\section{Materials and Methods}

\section{Sample Preparation}

The specimens were obtained from bovine incisor crowns and adapted on a cutting machine (model ELSAW, ElQuip). With the aid of a diamond disc (model ER04003 HC $4 \times 0.012 \times 1 / 2$, ERIOS equipment), they were sectioned with the crown separated from the root of the dental units. Buccolingual cuts were made to obtain 80 fragments of $8 \times 8 \times 2 \mathrm{~mm}$ in size, which were flattened for standardization of the surfaces in a PL VO60 (Biopdi; São Carlos, SP, Brazil) with silicon carbide water sanding discs of 180, 400, and 600 grit (3M Company, Brasil Ltda). The granulations by Caldeira et $\mathrm{al}^{10}$ and from the recommendations of ISO/TS $11405^{11}$ were used to plan the bonding area. After polishing, they were fixed in orthophthalic resin, placed in an L-200 ultrasonic vat (Schuster Ltda.) for 10 minutes for cleaning and organized into experimental groups according to the selected dentifrice ( $\mathbf{- T a b l e ~} \mathbf{1}$ ). After the experimental period, the specimens were evaluated in terms of whitening action by the dentrifrices, shear strength and adhesive remnant index (ARI) (-Fig. 1).

For this study, the sample calculation was performed in the Gpower 1 and R2 programs, based on the effect sizes found in the literature ${ }^{12,13}$ and ISO/TS 11405 recommendations for study design. ${ }^{11}$ Thus, the sample size of 80 dental units ( $n=20$ /group) provided a power of 0.80 for a significance level of $5 \%$.

\section{Orthodontics Brackets Bonding}

The specimens were cleaned according to the manufacturer's recommendations (3M Company; St. Paul, MN, United States). Subsequently, 37\% phosphoric acid conditioner gel was applied to the dental surfaces for 30 seconds, which were then rinsed with water and air dried. A uniform layer of primer was applied to the tooth surfaces, and Transbond XT adhesive (3M Company; St. Paul, MN, USA) was applied to the base of the bracket positioned on the tooth surface. The Transbond XT bracket-bonding adhesive system (3M Company) was chosen because it has lower TEGDMA release and is considered the gold standard in orthodontics. ${ }^{14-17}$ Excess material was removed, and the surfaces were light cured (DB 686 Wireless Dabi Atlante) at a distance of 2 to $3 \mathrm{~mm}$ for 10 seconds on each interproximal face. ${ }^{10}$

\section{Dentifrices Solutions}

The dentifrices were weighed on an AY 220 precision scale (Shimadzu Ltda.), diluted 1:2 in deionized water, and subjected to $\mathrm{pH}$ verification (Model 2000 Quimis Apparatus, Científicos Ltda.) after calibration in triplicate. ${ }^{18}$

\section{Simulated Brushing Abrasion Test}

Fifty thousand simulated brushing cycles were performed, which corresponds to one year of brushing. ${ }^{18}$ The speed of the simulated brushing machine (ElQuip) was 4.5 cycles/second in 10 back-and-forth arm movements. Each specimen was positioned on the machine by group, with a pre-fitted brush (Slim Soft Black, Colgate, Colgate-Palmolive Co, ltda.) and a 20-ml syringe that injected $0.4 \mathrm{ml}$ of the solution every 2 minute. ${ }^{18}$

\section{Color Analysis}

The Easyshade Vita spectrophotometer provides readings on the CIE L*a*b* system, in which colors are defined in three parameters: L *-brightness, which ranges from 0 to 100; $a$ *-red-green, ranging from -80 to +80 ; and $b$ *-blue yellow, ranging from -80 to +80 . This system also allows

Table 1 Selected dentifrices' composition and manufacturer

\begin{tabular}{|c|c|c|c|}
\hline Dentifrice & Principal composition & $\begin{array}{l}\text { Whitening } \\
\text { agents }\end{array}$ & Manufacturer \\
\hline Colgate Total 12 & $\begin{array}{l}0.32 \% \text { sodium fluoride (1,450 ppm fluoride), } 0.3 \% \text { triclosan, } \\
\text { water, hydrated silica }\end{array}$ & Mechanical & Colgate-Palmolive \\
\hline $\begin{array}{l}\text { Curaprox Black Is } \\
\text { White }\end{array}$ & $\begin{array}{l}\text { Water, sorbitol, hydrated silica, glycerin, activated charcoal, } \\
\text { aroma, bentonite, sodium monoflourophosphate, mica, cetearyl } \\
\text { alcohol, lemon CI 75815, Cl } 77289\end{array}$ & Mechanical & Curaden-Swiss \\
\hline $\begin{array}{l}\text { Luminous White } \\
\text { Advanced }\end{array}$ & $\begin{array}{l}2 \% \text { hydrogen peroxide, } 0.76 \% \text { sodium monofluorophos- } \\
\text { phate, propylene glycol, calcium pyrophosphate, glycerine, } \\
2 \% \text { polyvinylpyrrolidone-hydrogen peroxide, silica }\end{array}$ & $\begin{array}{l}\text { Mechanical and } \\
\text { chemical }\end{array}$ & Colgate-Palmolive \\
\hline
\end{tabular}




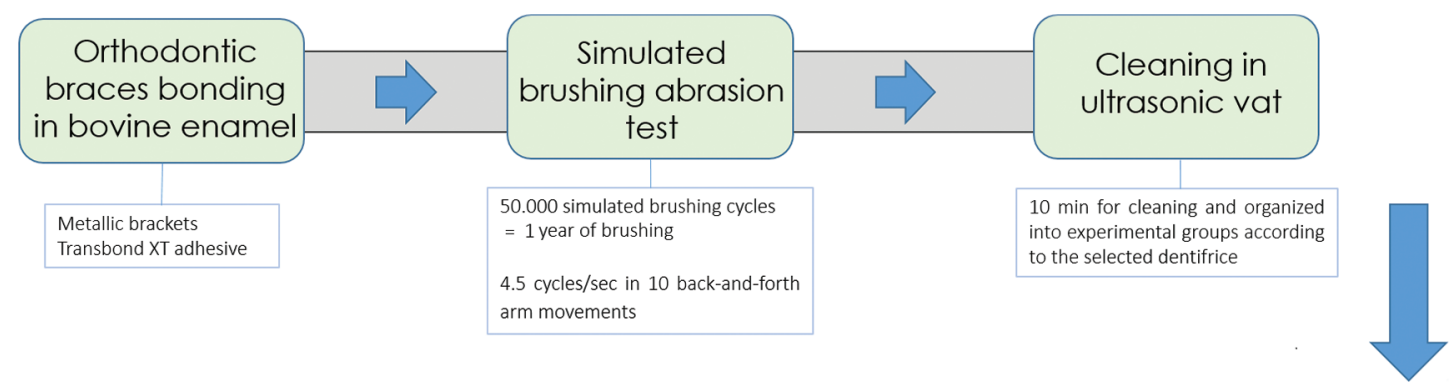

a

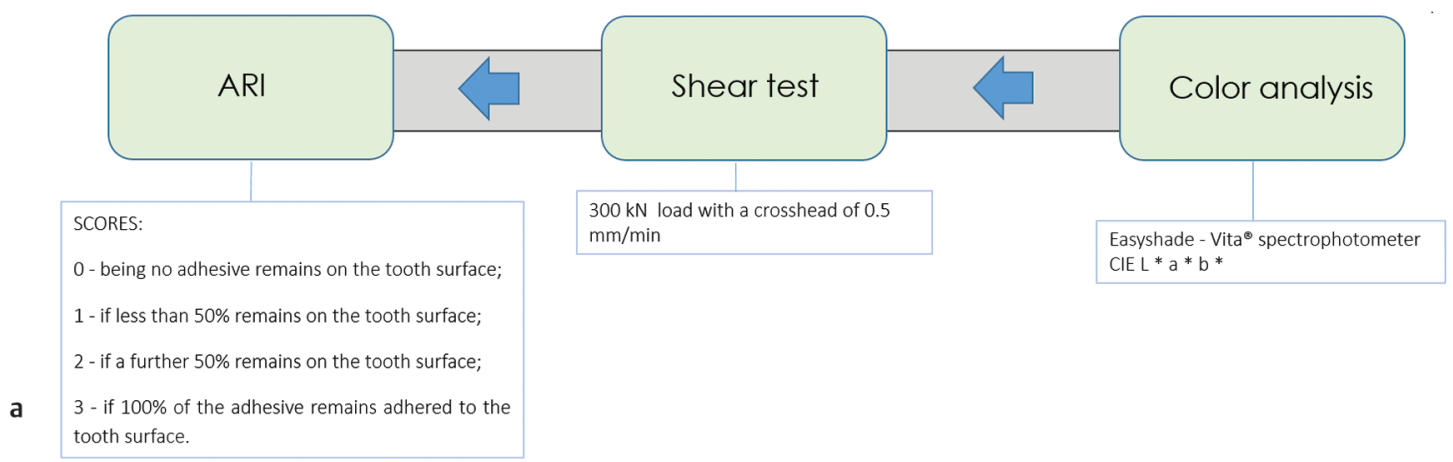

Fig. 1 Experimental design. ARI, adhesive remnant index.

the color difference between two samples to be measured $(\Delta \mathrm{E}-\Delta \mathrm{E})$ and demonstrates the amount of color change between two readings. The color parameters were obtained before and at 6 and 12 months of simulated brushing. ${ }^{19,20}$

\section{Shear Test and Adhesive Remnant Index}

The shear test was performed in a universal testing machine (Model DL 23-300; EMIC - Instron Brazil) using a 300 kN load with a crosshead of $0.5 \mathrm{~mm} / \mathrm{min}$.

The enamel surface and support base of each tooth were examined for remnant adhesive. The ARI is an index proposed by Artun and Bergland ${ }^{21}$ with scores from 0 to 3, with 0 being when no adhesive remains on the tooth surface; 1 if less than $50 \%$ remains on the tooth surface; 2 if a further $50 \%$ remains on the tooth surface; or 3 if $100 \%$ of the adhesive remains adhered to the tooth surface with a visible supportive impression.

\section{Statistical Analysis}

The maximum force in $\mathrm{N}$ was converted to Mpa. The maximum force data were submitted to one-way analysis of variance (ANOVA) and Tukey's test. Kruskal-Wallis and Dunn's nonparametric tests were used for color analysis to compare groups, and Friedman and Nemenyi tests were used to compare times. ARI analysis was performed with Fisher's exact test. All analyses were performed using the R program, with a significance level of $5 \%$.

\section{Results}

Shear strength, ARI, and color variation were analyzed. GT1 presented significantly higher shear force than GT3 $(p \leq 0.05)$.
Table 2 Average (pattern deviation) of maximum shear force (N) as a function of group

\begin{tabular}{|l|l|}
\hline Group & Maximum shear force \\
\hline Water & $190.89(107.12)^{\mathrm{a}}$ \\
\hline Colgate Total 12 & $208.35(99.68)^{\mathrm{a}}$ \\
\hline Curaprox Black Is White & $165.75(110.48)^{\mathrm{a}, \mathrm{b}}$ \\
\hline Luminous White Advanced & $126.20(90.24)^{\mathrm{b}}$ \\
\hline
\end{tabular}

Note: Superscript letters show difference between the groups with significance level of $5 \%(p<0.05)$.

The other groups did not differ in maximum strength $(p>0.05)$. GT3 presented lower shear force ( - Table 2$)$. In the CG, only $5 \%$ of the specimens had $100 \%$ adhesive on the dental surface. In the experimental groups, this percentage was $15 \%$ in GT1, 40\% in GT2, and 45\% in GT3. In the CG, $90 \%$ of the specimens had between 0 and $50 \%$ adhesive on the dental surface. The experimental groups had $70 \%$ for GT1, $45 \%$ for GT2, and 45\% for GT3 (-Fig. 2).

At 6 months of brushing, the $L$ value increased significantly ( $p \leq 0.05$ ) for all groups except for GT1 ( $p \leq 0.05)$. The value of $a$ decreased significantly in all groups ( $p \leq 0.05)$. The value of $b$ significantly decreased in GT2 and GT3 ( $p \leq 0.05)$. The total color variation $(\Delta \mathrm{E})$ was significantly higher in GT3 than in the other groups $(p \leq 0.05)$. At 12 months, the value of $L$ was significantly higher in all four groups than in the initial evaluation ( $p \leq 0.05)$. The value of $L$ was significantly higher in GT3 than in GT1 and CG ( $p \leq 0.05)$. In all four groups, the value of $a$ was significantly lower than at baseline ( $p \leq 0.05)$. In GT2 and GT3, the value of $b$ was significantly lower than in the initial evaluation ( $p \leq 0.05$ ). Lastly, $\Delta \mathrm{E}$ was significantly higher in GT3 than in CG and GT1 ( $p \leq 0.05)$ (-Fig. 3). 


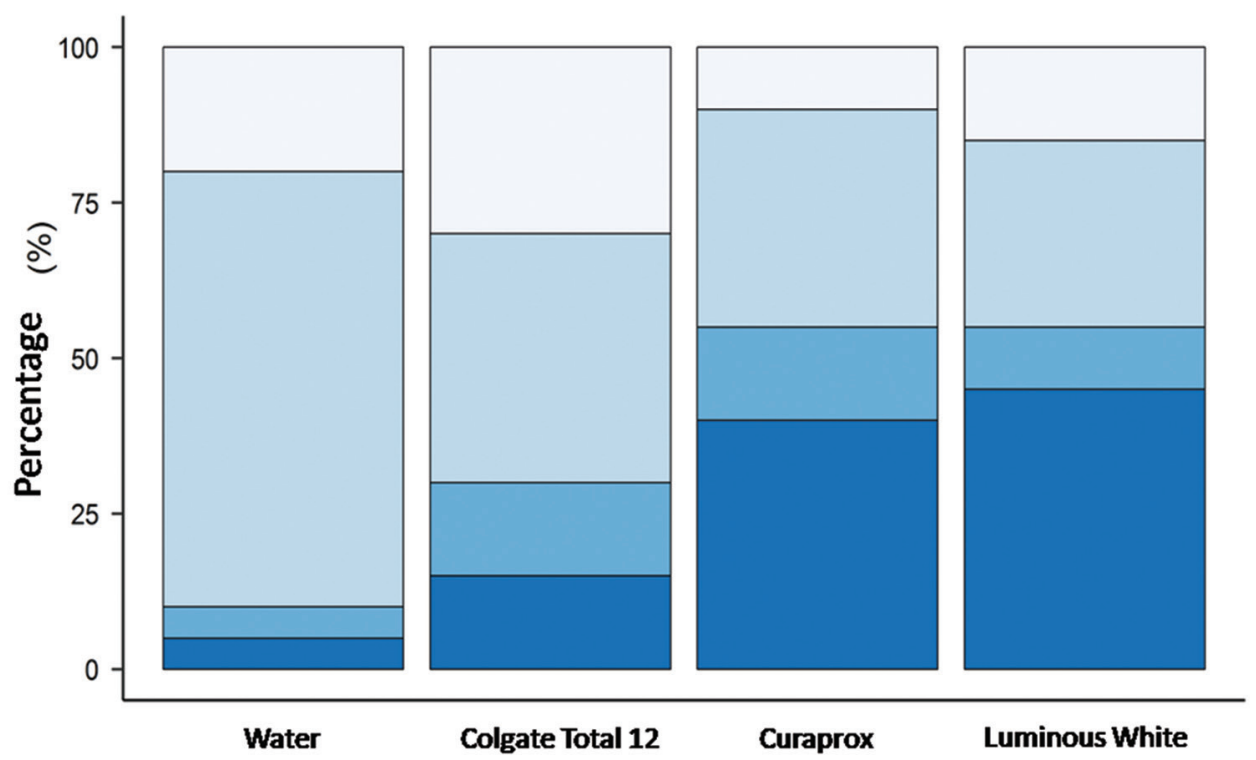

ARI $\square 0 \square 1 \square 2 \square 3$

Fig. 2 Specimen distribution in each group as a function of adhesive remnant index. ARI, adhesive remnant index.

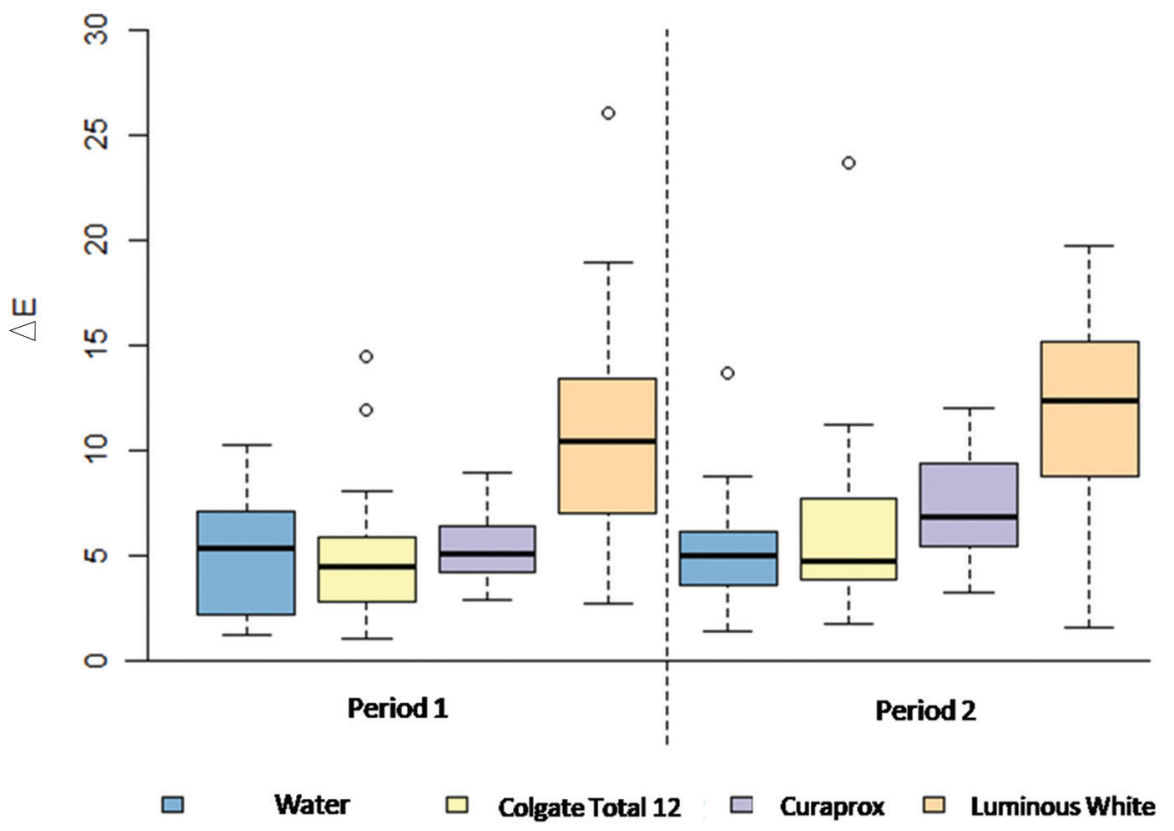

Fig. 3 Box plot of the value $(\Delta E)$ as a function of group and time. Period 1: 6 initial brushing months, period 2: 12 initial brushing months.

\section{Discussion}

The use of whitening dentifrices during orthodontic treatment may interfere with the brackets' adhesion and consequently in the instituted therapy, as well as produce alterations to abrasiveness and color in the dental enamel, altering the aesthetics at the end of the treatment. Thus, the present study evaluated the interference of these whitening agents in the resistance to orthodontic bonding and the abrasiveness and color of the enamel after detachment of the brackets.
Commercially available products may have whitening properties and remove extrinsic stains from the dental surface, such as silica and activated charcoal. On the other hand, bleaching agents such as $\mathrm{H}_{2} \mathrm{O}_{2}$ change the intrinsic color of the dentin and enamel in a deeper and more lasting way. ${ }^{22} \mathrm{~A}$ wide variety of whitening dentifrices are available in the market, and their main action is through mechanical removal of acquired film and extrinsic stains and polishing of the enamel surface. ${ }^{2}$ Some of these products with bleaching agents have low concentrations of $\mathrm{H}_{2} \mathrm{O}_{2}$, in an attempt to improve abrasive cleaning, to help remove extrinsic stains. ${ }^{23,24}$ 
Another abrasive agent, activated charcoal, may be added to a dentifrice's formulation to promote whitening. However, there is no evidence that dental enamel damage can occur. ${ }^{25}$ Patients should be directed to use these formulations properly, as there may be potential for increased abrasiveness and damage to enamel. ${ }^{4,22}$

Whitening dentifrices can be more effective in altering the color of teeth than the conventional dentifrices. The best whitening performance was obtained in microsphere dentifrices, followed by those with hydrogen peroxide and blue covarine dye (CI74160), ${ }^{4,12}$ These results corroborate the present study, in which groups containing abrasive agents such as activated charcoal or bleaching agents such as hydrogen peroxide showed significant color change over the initial 6 months and progressive change over the final 6 months. $\mathrm{H}_{2} \mathrm{O}_{2}$ showed a higher perception of whiteness compared with the other groups. The group with activated charcoal also showed significant color change, and the presence of bright microspheres during the study may suggest an optical effect besides the mechanical whitening effect. In this same group, at the end of the 12 months of simulated brushing, a whitening effect was observed in $\mathrm{H}_{2} \mathrm{O}_{2}$ group. The silica and water groups had the lowest color variation values.

The American Dental Association considers bleaching effective when $\Delta \mathrm{E}$ is at least $3 .^{26}$ In the present study, this parameter was greater than 3 in all of the analyzed periods, demonstrating that whitening was effective after 6 and 12 months of brushing in all of the analyzed groups.

Microleakages can be observed at the interfaces of orthodontic brackets bonded to different adhesive systems. ${ }^{27}$ The brushing abrasion with whitening or bleaching agents presents in dentifrices can promote greater enamel wear than the conventional dentifrices. ${ }^{28}$ Such condition may have favored microleakage and interfered with the adhesion of brackets to the enamel surface. In the present study, silica group presented higher shear strength than the other groups, and $\mathrm{H}_{2} \mathrm{O}_{2}$ group presented lower resistance. Reduced bracket bond strength in bleached teeth has been related to changes in enamel mineral and protein content and not to the effects of residual oxygen. ${ }^{29}$

According to the results obtained in our study, the activated charcoal and $\mathrm{H}_{2} \mathrm{O}_{2}$ groups presented an ARI of around $45 \%$ with score 3 , thus suggesting some interference in the adhesive-base mechanical adhesion of the bracket submitted to the activated charcoal agent or $\mathrm{H}_{2} \mathrm{O}_{2}$ bleaching agent. The hardness, shape, size, and concentration of particles in dentifrices influence their abrasiveness. ${ }^{30} \mathrm{H}_{2} \mathrm{O}_{2}$ dentifrices and activated carbon seems to have influenced the reduction of bond strength of the metal brackets. Due to their abrasive and high-dissolution effects and fluidity when present in dentifrices, these substances may interfere with orthodontic adhesion..$^{31}$ Thus, it is hoped that the results of the present study can positively inform and influence the guidance given to patients. The professional has an important role in indicating the most suitable dentifrice for each need once in vitro studies are similar to those in vivo. ${ }^{32}$

\section{Conclusion}

Simulated brushing with whitening dentifrices containing mechanical and chemical agents was effective in modifying the visual perception of the color of bovine enamel; however, the dentifrices containing the oxygen peroxide agents and activated charcoal seems to have negatively influenced the shear bond strength.

\section{Conflict of Interest}

None declared.

\section{Acknowledgments}

The authors would like to thank the Health Sciences Institute of UFBA - Biochemistry and Biophysics Department; Federal Institute of Bahia-Destructive Testing Laboratory/ IFBA and Hermínio Ometto Foundation/FHO for their assistance in this study.

\section{References}

1 Vahid Dastjerdi E, Khaloo N, Mojahedi SM, Azarsina M. Shear bond strength of orthodontic brackets to tooth enamel after treatment with different tooth bleaching methods. Iran Red Crescent Med J 2015;17(11):e20618

2 Li Y. Stain removal and whitening by baking soda dentifrice: a review of literature. J Am Dent Assoc 2017;148(11S):S20-S26

3 Bizhang M, Chun YH, Damerau K, Singh P, Raab WH, Zimmer S Comparative clinical study of the effectiveness of three different bleaching methods. Oper Dent 2009;34(6):635-641

4 Vaz VTP, Jubilato DP, Oliveira MRM, et al. Whitening toothpaste containing activated charcoal, blue covarine, hydrogen peroxide or microbeads: which one is the most effective? J Appl Oral Sci 2019;27:e20180051

5 van Loveren C, Duckworth RM. Anti-calculus and whitening toothpastes. Monogr Oral Sci 2013;23:61-74

6 Alshara S, Lippert F, Eckert GJ, Hara AT. Effectiveness and mode of action of whitening dentifrices on enamel extrinsic stains. Clin Oral Investig 2014;18(2):563-569

7 Watts A, Addy M. Tooth discolouration and staining: a review of the literature. Br Dent J 2001;190(6):309-316

8 Walsh TF, Rawlinson A, Wildgoose D, Marlow I, Haywood J, Ward JM. Clinical evaluation of the stain removing ability of a whitening dentifrice and stain controlling system. J Dent 2005;33(5):413-418

9 Iska D, Devanna R, Singh M, Chitumalla R, Balasubramanian $\mathrm{SCB}$, Goutam M. In vitro assessment of influence of various bleaching protocols on the strength of ceramic orthodontic brackets bonded to bleached tooth surface: a comparative study. J Contemp Dent Pract 2017;18(12):1181-1184

10 Caldeira EM, Fidalgo TK, Passalini P, Marquezan M, Maia LC, Nojima MdaC. Effect of fluoride on tooth erosion around orthodontic brackets. Braz Dent J 2012;23(5):581-585

11 International Organization for Standardization ISO/TS 11405:2015. Dentistry - testing of adhesion to tooth structure. Available at: https://www.iso.org/standard/62898.html. Accessed December 27, 2019

12 Bergesch V, Baggio Aguiar FH, Turssi CP, Gomes França FM, Basting RT, Botelho Amaral FL. Shade changing effectiveness of plasdone and blue covarine-based whitening toothpaste on teeth stained with chlorhexidine and black tea. Eur J Dent 2017;11(4):432-437 
13 Yadav D, Golchha V, Paul R, Sharma P, Wadhwa J, Taneja S. Effect of tooth bleaching on orthodontic stainless steel bracket bond strength. J Orthod Sci 2015;4(3):72-76

14 Pelourde C, Bationo R, Boileau MJ, Colat-Parros J, Jordana F. Monomer release from orthodontic retentions: an in vitro study. Am J Orthod Dentofacial Orthop 2018;153(2):248-254

15 Wongsamut W, Satrawaha S, Wayakanon K. Surface modification for bonding between amalgam and orthodontic brackets. J Orthod Sci 2017;6(4):129-135

16 Seeliger JH, Botzenhart UU, Gedrange T, Kozak K, Stepien L, Machoy M. Enamel shear bond strength of different primers combined with an orthodontic adhesive paste. Biomed Tech (Berl) 2017;62(4):415-420

17 Northrup RG, Berzins DW, Bradley TG, Schuckit W. Shear bond strength comparison between two orthodontic adhesives and self-ligating and conventional brackets. Angle Orthod 2007;77(4):701-706

18 Odilon NN, Lima MJP, Ribeiro PL, Araújo RPCde, Campos Ede J. In vitro evaluation of the effect of bleaching dentifrices containg blue covarine on bovine dental enamel. Rev Odontol UNESP 2018;47:388-394

19 Tao D, Smith RN, Zhang Q, et al. Tooth whitening evaluation of blue covarine containing toothpastes. J Dent 2017;67S:S20-S24

20 Kalantari MH, Ghoraishian SA, Mohaghegh M. Evaluation of accuracy of shade selection using two spectrophotometer systems: Vita Easyshade and Degudent Shadepilot. Eur J Dent 2017;11(2):196-200

21 Artun J, Bergland S. Clinical trials with crystal growth conditioning as an alternative to acid-etch enamel pretreatment. Am J Orthod 1984;85(4):333-340

22 Greenwall LH, Greenwall-Cohen J, Wilson NHF. Charcoal-containing dentifrices. Br Dent J 2019;226(9):697-700
23 Pintado-Palomino K, Vasconcelos CV, Silva RJ, et al. Effect of whitening dentifrices: a double-blind randomized controlled trial. Braz Oral Res 2016;30(1):e82

24 Vieira GH, Nogueira MB, Gaio EJ, Rosing CK, Santiago SL, Rego RO. Effect of whitening toothpastes on dentin abrasion: an in vitro study. Oral Health Prev Dent 2016;14(6):547-553

25 Brooks JK, Bashirelahi N, Reynolds MA. Charcoal and charcoal-based dentifrices: a literature review. J Am Dent Assoc 2017;148(9):661-670

26 Siew C; American Dental Association. ADA guidelines for the acceptance of tooth-whitening products. Compend Contin Educ Dent Suppl 2000;28(28):S44-S47

27 Atash R, Fneiche A, Cetik S, et al. In vitro evaluation of microleakage under orthodontic brackets bonded with different adhesive systems. Eur J Dent 2017;11(2):180-185

28 Ionta FQ, Dos Santos NM, Mesquita IM, et al. Is the dentifrice containing calcium silicate, sodium phosphate, and fluoride able to protect enamel against chemical mechanical wear? An in situ/ex vivo study. Clin Oral Investig 2019;23(10):3713-3720

29 Perdigão J, Francci C, Swift EJ Jr, Ambrose WW, Lopes M. Ultra-morphological study of the interaction of dental adhesives with carbamide peroxide-bleached enamel. Am J Dent 1998;11(6):291-301

30 Joiner A. Whitening toothpastes: a review of the literature. J Dent 2010;38(Suppl 2):e17-e24

31 Britto FA, Lucato AS, Valdrighi HC, Vedovello SAS. Influence of bleaching and desensitizing gel on bond strength of orthodontic brackets. Dental Press J Orthod 2015;20(2):49-54

32 Ahmed T, Rahman NA, Alam MK. Assessment of in vivo bond strength studies of the orthodontic bracket-adhesive system: a systematic review. Eur J Dent 2018;12(4):602-609 